\title{
RANDOM RECURSIVE CONSTRUCTIONS: ASYMPTOTIC GEOMETRIC AND TOPOLOGICAL PROPERTIES
}

\author{
R. DANIEL MAULDIN ${ }^{1}$ AND S. C. WILLIAMS
}

\begin{abstract}
We study some notions of "random recursive constructions" in Euclidean $m$-space which lead almost surely to a particular type of topological object; e.g., Cantor set, Sierpiński curve or Menger curve. We demonstrate that associated with each such construction is a "universal" number $\alpha$ such that almost surely the random object has Hausdorff dimension $\alpha$. This number is the expected value of the sum of some ratios which in the deterministic case yields Moran's formula.
\end{abstract}

We introduce the notion of a "random recursive construction" and prove several basic facts about such constructions. We give specific examples which lead to random Cantor sets, Sierpiński curves or Menger universal curves. It is perhaps best to begin with a specific example of such a construction. To this end, let us make some notation. Let $\mathbf{N}$ be the set of positive integers and $\mathbf{R}$ the real numbers. If $S$ is a set, let $S^{*}$ be the set of all finite sequences of elements of $S$ including $\varnothing$, the empty sequence. If $\alpha=\left(a_{1}, \ldots, a_{n}\right)$ and $\beta=\left(b_{1}, \ldots, b_{m}\right)$ are elements of $S$, then $|\alpha|=n$, the length of $\alpha$, and $\alpha * \beta=\left(a_{1}, \ldots, a_{n}, b_{1}, \ldots, b_{m}\right)$. Now, consider the following construction of a Cantor subset of $[0,1]$, the unit interval. (Of course, by a Cantor set we mean a compact, perfect, 0-dimensional metric space.) Set $J_{\varnothing}=[0,1]$ and, by recursion, if $J_{\sigma}=[a, b]$, for $\sigma \in\{0,1\}^{*}$, then set $J_{\sigma * 0}=[a, a+x(b-a)]$ and $J_{\sigma * 1}=[a+y(b-a), b]$, where the point $(x, y)$ is chosen from the triangular region $\Delta=\{(s, t) \mid 0 \leq s \leq t \leq 1\}$ according to the uniform distribution. It follows from the results given in this paper that with probability one, the set

$$
K=\bigcap_{n}\left[\bigcup_{\sigma \in\{0,1\}^{n}} J_{\sigma}\right]
$$

is a Cantor set and the Hausdorff dimension of $K, \operatorname{dim}_{H}(K)$, is $(\sqrt{17}-3) / 2$.

The paper is organized into four sections. In $\S 1$, we define the notion of a random construction and prove a few basic facts concerning such a construction. We demonstrate that with each construction there is a number $\alpha$ such that with probability one the object constructed has Hausdorff dimension $\leq \alpha$. In this section, we relate our results to some deterministic results of P. A. P. Moran [15].

In $\S 2$ (Theorem 2.1 ), we show that certain commonly occurring constructions have finite moments of all orders. This result is necessary for our proof that with probability one the Hausdorff dimension is $\alpha$.

Received by the editors December 27, 1984 and, in revised form, July 9, 1985.

1980 Mathematics Subject Classification. Primary 54H20, 60B05; Secondary 28C10.

Key words and phrases. Martingale, Hausdorff dimension, Cantor set, Sierpiński curve, Menger curve.

${ }^{1}$ Research supported in part by a grant from the National Science Foundation. 
In $\S 3$, we construct a random measure associated with the construction. For each object $K_{\omega}$ generated by the construction there corresponds a Borel measure $\nu_{\omega}$ supported on $K_{\omega}$ such that if $\beta<\alpha$ and $E$ is a set with finite $\beta-m$ measure, then $\nu_{\omega}(E)=0$. More accurately, in Theorem 3.6, we show that there is a sequence of measures $\nu_{\omega, n}$ supported by $K_{\omega}$ such that if $\beta<\alpha$ and $\beta-m(E)<\infty$, then there is some $n_{0}$ with $\nu_{\omega, n_{0}}\left(K_{\omega}\right)>0$ and $\nu_{\omega, n_{0}}(E)=0$. This, of course, implies that $\operatorname{dim}_{H}\left(K_{\omega}\right)=\alpha$. Our methods involve a probabilistic mixture of some deterministic results of Moran and of Rogers and Taylor [15, 16]. We remark that there is at least one major unsolved problem in this context. The problem is that we do not have conditions under which one can be guaranteed that with probability one, $\alpha-m\left(K_{\omega}\right)>0$, although this seems to be the case with many constructions. We comment on this at the end of $\S 3$. By $\alpha-m(E)$, we mean the measure of $E$ with respect to the Hausdorff measure defined by the function $h(x)=x^{\alpha}$.

In the fourth and last section, we give a number of specific examples of constructions which we hope illustrate some of the possibilities. We give several examples of random Cantor subsets of $[0,1]$. In Example 4.6, we construct random Cantor subsets of $[0,1] \times[0,1]$ with Hausdorff dimension 1. In Example 4.7 we generate random Sierpiński curves. In Example 4.8 we generate random locally connected nonplanar continua. We are unable at this time to show that they are Menger curves. In Example 4.9 we modify the construction to generate random Menger curves.

Our construction and the results we prove have features in common with several other processes of current interest, and we believe our methods may be useful in their development. For example, our construction incorporates two features common to the theses of Mandelbrot $[\mathbf{1 1}, \mathbf{1 2}]$. It certainly maintains a degree of randomness and yet at the same time preserves some properties of self-similarity. Perhaps even closer to the heart of the matter is the definition of fractal geometry and a fractal given by Cannon [4]. A random construction codifies certain geometric-algorithmic processes which by their nature exhibit some random behavior. The examples given in the last section indicate what one can say about the asymptotic geometric or topological shape of particular constructions. Falconer gives a more complete listing of recent references and a development of some of the central general issues [7]. Related topics are treated by Zähle [19].

1. Random constructions and the $\Phi$-function. Our general model is as follows: We fix a Euclidean space $\mathbf{R}^{m}$ and a nonempty compact subset $J$ of $\mathbf{R}^{m}$. We further require that $J$ is the closure of its interior in $\mathbf{R}^{m}$. We assume we have a probability space $(\Omega, \Sigma, P)$ and are given a family of random subsets of $\mathbf{R}^{m}$,

$$
\mathbf{J}=\left\{J_{\sigma} \mid \sigma \in \mathbf{N}^{*}=\bigcup_{n=0}^{\infty} \mathbf{N}^{n}\right\},
$$

satisfying three properties.

(1) $J_{\varnothing}(\omega)=J$ for almost all $\omega \in \Omega$. For every $\sigma \in \mathbf{N}^{*}$ and for almost all $\omega$, if $J_{\sigma}(\omega)$ is nonempty, then $J_{\sigma}(\omega)$ is geometrically similar to $J$.

(2) For almost every $\omega$ and for every $\sigma \in \mathbf{N}^{*}, J_{\sigma * 1}(\omega), J_{\sigma * 2}(\omega), J_{\sigma * 3}(\omega), \ldots$ is a sequence of nonoverlapping subsets of $J_{\sigma}(\omega)$. ( $A$ and $B$ nonoverlapping means $\operatorname{int} A \cap \operatorname{int} B=\varnothing$.) 
(3) The random vectors $\tau_{\sigma}=\left\langle T_{\sigma * 1}, T_{\sigma * 2}, \ldots\right\rangle, \sigma \in \mathbf{N}^{*}$, are i.i.d., where $T_{\sigma * n}(\omega)$ equals the ratio of the diameter of $J_{\sigma * n}(\omega)$ to the diameter of $J_{\sigma}(\omega)$ if $J_{\sigma}(\omega)$ is nonempty. (For convenience, let $T_{\varnothing}(\omega)=$ diameter of $J$.)

We shall call such a system $\mathbf{J}$ a construction. Our constructions require only a "stochastic ratio self-similarity". We now define the random set $K$ by

$$
K(\omega)=\bigcap_{n=1}^{\infty} \bigcup_{\sigma \in \mathrm{N}^{n}} J_{\sigma}(\omega) .
$$

Our interest centers on the asymptotic properties of this random set $K$.

For convenience, let $0^{0}=0$. Then $\sum_{p=1}^{\infty} T_{\sigma * p}^{0}(\omega)$ counts the number of nonempty $J_{\sigma * p}(\omega)$, if $J_{\sigma}(\omega)$ is itself nonempty.

THEOREM 1.1. Suppose $E\left(\sum_{n=1}^{\infty} T_{n}^{0}\right)>1$. Then, with positive probability, $K$ is nonempty. Moreover, given that $K$ is nonempty, then almost surely $K$ has Hausdorff dimension $\alpha$, where $\alpha$ is the least $\beta>0$ such that $E\left(\sum_{n=1}^{\infty} T_{n}^{\beta}\right) \leq 1$.

We shall establish this theorem by proving several simpler theorems. But first we shall show that $\alpha$ is well defined, relate our results to some results of P. A. P. Moran [15], and motivate the hypothesis $E\left(\sum_{n=1}^{\infty} T_{n}^{0}\right)>1$.

Let $\lambda$ denote $m$-dimensional Lebesgue measure and define $\Phi:[0, \infty) \rightarrow[0, \infty]$ by

$$
\Phi(\beta)=E\left(\sum_{n=1}^{\infty} T_{n}^{\beta}\right)=E\left(\sum_{n=1}^{\infty} T_{\sigma * n}^{\beta}\right),
$$

where $\sigma$ can be any member of $\mathbf{N}^{*}$. Now, using the monotone convergence theorem and the fact $T_{i} \in[0,1]$ for each $i$, we see that $\Phi$ is nonincreasing and right continuous. We have

$$
\sum_{n=1}^{\infty} \lambda\left(\operatorname{int}\left(J_{n}\right)\right) \leq \lambda(\operatorname{int}(J))
$$

and by the scaling property of $\lambda$,

$$
\lambda\left(\operatorname{int}\left(J_{n}\right)\right)=T_{n}^{m} \lambda(\operatorname{int}(J)) .
$$

Thus,

$$
\sum_{n=1}^{\infty} T_{n}^{m} \leq 1 \quad \text { a.s. }
$$

Therefore, $\Phi(m) \leq 1$. From these facts it follows that $\alpha$ is well defined. (An example with $\alpha=m$ is given in Example 4.5.)

Concerning the calculation of $\alpha$, note if $\Phi(y)<\infty$, then $\Phi$ is continuous on $[y,+\infty)$ and if, in addition, $\Phi(0)>1$, then $\Phi$ is strictly decreasing on $[y, \infty)$. Thus, if $\Phi(0)>1$ and $\Phi(\beta)=1$, then $\beta=\alpha$. However, as Example 4.5 shows, in the general case, $\Phi(\alpha)$ may be less than 1 .

An interesting special case of our construction occurs on a $n$-ary tree; i.e., for some fixed $n \in \mathbf{N}$, our construction is

$$
\mathbf{J}=\left\{J_{\sigma} \mid \sigma \in\{1,2, \ldots, n\}^{*}=\bigcup_{j=1}^{\infty}\{1,2, \ldots, n\}^{j}\right\},
$$


or what is essentially the same in our construction using $\mathrm{N}^{*}$, we have $\sum_{k=1}^{\infty} T_{k}^{0} \leq n$ a.s. In this case, $\Phi$ is a finite valued continuous function, and if further $\Phi(0)>1$, then $\Phi(\beta)=1$ has a solution, and so trivially $\Phi(\alpha)=1$. This will be used later.

As a further special case, if $\tau_{\varnothing}$ is distributed as point mass at $\left(t_{1}, t_{2}, \ldots, t_{n}, 0,0\right.$, $0, \ldots)$, where $t_{1}, t_{2}, \ldots, t_{n}>0$, Theorem 1.1 implies $K$ has Hausdorff dimension $\alpha$ where $\alpha$ satisfies the equation $t_{1}^{\alpha}+t_{2}^{\alpha}+\cdots+t_{n}^{\alpha}=1$. This result was proved by $\mathrm{P}$. A. P. Moran in [15]. In this sense, $\alpha=\min \left\{\beta>0 \mid E\left(T_{1}^{\beta}+T_{2}^{\beta}+\cdots\right) \leq 1\right\}$ and $E\left(T_{1}^{\alpha}+T_{2}^{\alpha}+\cdots\right)=1$, in the special case when $\Phi$ is continuous, are generalizations of Moran's equation.

For each $\sigma \in \mathbf{N}^{*}$, we define the random variable $l_{\sigma}$ by

$$
l_{\sigma}=\operatorname{diameter}\left(J_{\sigma}\right)=\prod_{n=0}^{|\sigma|} T_{\sigma \mid n},
$$

where $|\sigma|$ is the length of the finite sequence $\sigma$ and $\sigma \mid n$ is the sequence obtained by restricting $\sigma$ to its first $n$ terms. $(\sigma \mid 0=\varnothing=$ empty sequence.) For each $n \in \mathbf{N}$, we let $\mathcal{F}_{n}$ denote the $\sigma$-algebra $\sigma-\left(\left\{T_{\gamma}:|\gamma| \leq n\right\}\right)=\sigma-\left(\left\{\tau_{\gamma}:|\gamma|<n\right\}\right)$. For each $n \in \mathbf{N}$ and $\beta \geq 0, S_{\beta, n}$ is the random variable

$$
S_{\beta, n}=\sum_{\sigma \in \mathbf{N}^{n}} l_{\sigma}^{\beta}
$$

We note the useful fact concerning conditional expectations:

$$
E\left(S_{\beta, n+1} \mid F_{n}\right)=\Phi(\beta) S_{\beta, n},
$$

where $\infty \cdot 0=0$. In order to derive (1.8), we use (1.7) and (1.6) to obtain the formula

$$
S_{\beta, n+1}=\sum_{\sigma \in \mathbb{N}^{n}} l_{\sigma}^{\beta}\left[\sum_{p=1}^{\infty} T_{\sigma * p}^{\beta}\right] .
$$

We obtain (1.8) by taking conditional expectations on both sides of (1.9), noting that $l_{\sigma}^{\beta}$ is $\mathcal{F}_{n}$-measurable for $|\sigma| \leq n$ and noting

$$
E\left(\sum_{p=1}^{\infty} T_{\sigma * p}^{\beta} \mid F_{n}\right)=E\left(\sum_{p=1}^{\infty} T_{\sigma * p}^{\beta}\right)=\Phi(\beta)
$$

since $\tau_{\sigma}$ is independent of $\xi_{n}$ for $n=|\sigma|$.

Now, we shall motivate the hypothesis $\Phi(0)>1$.

Clearly, $S_{0, n}$ counts the number of nonempty $J_{\sigma}$ for $\sigma \in \mathbf{N}^{n}$. In fact, $\left\{S_{0, n}\right\}_{n=1}^{\infty}$ is a classical Galton-Watson branching process, in case $P\left(\sum T_{n}^{0}<\infty\right)=1$ (see $[\mathbf{1}$, pp. 7-8]). Further, $\Phi(0)$ is the mean number of offspring of a single parent in the branching process. It is well known that if $\Phi(0) \leq 1$, then either $\left\{S_{0, n}\right\}_{n=1}^{\infty}$ is a process bound for extinction $\left(S_{0, n} \rightarrow 0\right.$ as $n \rightarrow \infty$ a.s.) or it is trivial $\left(S_{0, n}=1\right.$ a.s., for each $n$ ). Thus, if $\Phi(0) \leq 1$, either $K=\varnothing$ a.s. or $K$ is a point a.s. or $K=J$ a.s. On the other hand, if $\Phi(0)>1$, a slight generalization of the well-known result is $S=\lim S_{0, n}$ exists a.s. and $S \in\{0, \infty\}$ a.s.

If $\Phi(0)>1$, then $P(S=\infty)>0$. It is clear from what we have said that the only interesting case is $\Phi(0)>1$. This will be assumed throughout the remainder of the paper but often stated for emphasis. 
THEOREM 1.2. Suppose $\Phi(0)>1$. Then for almost all $\omega$,

$$
" S(\omega)=\infty \text { if and only if } K(\omega) \neq \varnothing " .
$$

Proof. Clearly, if $S(\omega) \neq \infty$, then almost surely $S(\omega)=0$. Thus, there is some $n \in \mathbf{N}$ such that $S_{0, n}(\omega)=0$. This means $K(\omega) \subseteq \bigcup_{\sigma \in \mathbb{N}^{n}} J_{\sigma}(\omega)=\varnothing$.

For the converse we consider two cases: Case A: $P\left(\sum_{n=1}^{\infty} T_{n}^{0}<\infty\right)=1$ and Case B: $P\left(\sum_{n=1}^{\infty} T_{n}^{0}=\infty\right)>0$.

Case A. Since $P\left(\sum_{n=1}^{\infty} T_{n}^{0}<\infty\right)=1$, then a.s., for each $n, \bigcup_{\sigma \in \mathbb{N}^{n}} J_{\sigma}(\omega)$ is a finite union of compact sets and thus is itself compact. Therefore, if $S(\omega)=\infty$, then $\bigcap_{n=1}^{\infty} \bigcup_{\sigma \in \mathbb{N}^{n}} J_{\sigma}(\omega) \neq \varnothing$, by the intersection property of a nested sequence of nonempty compact sets.

Case B. Suppose $P\left(\sum_{n=1}^{\infty} T_{n}^{0}=\infty\right)=\delta>0$. We claim that for any $\sigma$, the following statement is true for almost all $\omega$ :

$$
\begin{aligned}
& \text { "If } \sum_{n=1}^{\infty} T_{\sigma * n}^{0}(\omega)=\infty \text {, then there exists } k \in \mathbf{N} \\
& \text { such that } T_{\sigma * k}^{0}(\omega)=1 \text { and } \sum_{n=1}^{\infty} T_{\sigma * k * n}^{0}(\omega)=\infty . "
\end{aligned}
$$

To see this, let $A$ be the event $\sum_{n=1}^{\infty} T_{\sigma * n}^{0}=\infty, B_{k}$ the event $\forall n \leq k$, if $T_{\sigma * n}^{0}=1$, then $\sum_{j=1}^{\infty} T_{\sigma * n * j}^{0}<\infty$ and $B_{\infty}$ be the event $\forall n \in \mathrm{N}$, if $T_{\sigma * n}^{0}=1$, then $\sum_{j=1}^{\infty} T_{\sigma * n * j}^{0}<\infty$. Let $H$ consist of all finite subsets of $\mathbf{N}$ and $G_{k}$ be the random set $\left\{n \leq k \mid T_{\sigma * n}^{0}=1\right\}$. Thus,

$$
\begin{aligned}
P\left(A \cap B_{\infty}\right) & \leq P\left(A \cap B_{k}\right)=\sum_{D \in H} P\left(A \cap B_{k} \text { and } D=G_{k}\right) \\
& =\sum_{D \in H} P\left(A, D=G_{k}, \forall n \in D\left[\sum_{j=1}^{\infty} T_{\sigma * n * j}^{0}<\infty\right]\right),
\end{aligned}
$$

and, by independence,

$$
=\sum_{D \in H} P\left(A \text { and } D=G_{k}\right)(1-\delta)^{\# D},
$$

where \#E denotes the cardinality of $E$. Thus,

$$
P\left(A \cap B_{\infty}\right) \leq \sum_{i=1}^{\infty} P\left(A \text { and } \# G_{k}=i\right)(1-\delta)^{i} .
$$

Fix $p \in \mathbf{N}$. Then

$$
P\left(A \cap B_{\infty}\right) \leq \sum_{i=1}^{p} P\left(A \text { and } \# G_{k}=i\right)+(1-\delta)^{p+1} P\left(A \text { and } \# G_{k} \geq p+1\right)
$$

or,

$$
P\left(A \cap B_{\infty}\right) \leq P\left(A \text { and } \# G_{k} \leq p\right)+(1-\delta)^{p+1} .
$$

Letting $k \rightarrow \infty$, we obtain

$$
P\left(A \cap B_{\infty}\right) \leq(1-\delta)^{p+1} .
$$

Now, letting $p \rightarrow \infty$, we find $P\left(A \cap B_{\infty}\right)=0$ or $P\left(B_{\infty} \mid A\right)=0$. This yields (1.11). 
From (1.11), it is easy to see that the following statement is true almost surely:

(1.12) "If $J_{\sigma}(\omega) \neq \varnothing$ and $\sum_{n=1}^{\infty} T_{\sigma * n}^{0}(\omega)=\infty$, then there exists a sequence of natural numbers $k_{1}, k_{2}, k_{3}, \ldots$ which depend on $\omega$, so that $J_{\sigma * k_{1} * k_{2} * \cdots * k_{t}}(\omega) \neq \varnothing$ for each $t$. Thus, $\varnothing \neq \bigcap_{t=1}^{\infty} J_{\sigma * k_{1} * \cdots * k_{t}}(\omega) \subseteq$ $K(\omega) "$.

Thus, we only need to establish that

$$
P\left(\exists \sigma \in \mathbf{N}^{*}\left[J_{\sigma} \neq \varnothing \text { and } \sum_{n=1}^{\infty} T_{\sigma * N}^{0}=\infty\right] \mid S=\infty\right)=1,
$$

in order to complete the proof in Case B.

Let $A$ be the event $\left(\forall \sigma \in \mathbf{N}^{*}\left[J_{\sigma} \neq \varnothing \rightarrow \sum_{n=1}^{\infty} T_{\sigma * n}^{0}<\infty\right]\right)$. For each $k$, let $A(k)$ denote the event $\left(\forall \sigma \in \mathbf{N}^{k}\left[J_{\sigma} \neq \varnothing \rightarrow \sum_{n=1}^{\infty} T_{\sigma * n}^{0}<\infty\right]\right)$. Then, concerning indicator functions, we have $1_{A} \leq 1_{A(n)}$ and $E\left(1_{A(n)} \mid F_{n}\right)=(1-\delta)^{S_{0, n}}$. Now, $E\left(1_{A} \mid \mathcal{F}_{n}\right) \rightarrow 1_{A}$ as $n \rightarrow \infty$. But, also, $E\left(1_{A} \mid \mathcal{F}_{n}\right) \leq(1-\delta)^{S_{0, n}} \rightarrow(1-\delta)^{S}$ as $n \rightarrow \infty$ almost surely. Therefore, for almost all $\omega$, if $S(\omega)=\infty$, then $\omega \notin A$. Q.E.D.

To begin the demonstration of Theorem 1.1, we know, according to $(1.5), \Phi(m) \leq$ 1. Thus, if $\Phi(0)>1, E\left(S_{m+1, n}\right)=(\operatorname{diam}(J))^{m+1}\left(\Phi(m+1)^{n}\right)$ and $E\left(\sum_{n=1}^{\infty} S_{m+1, n}\right)$ $=(\operatorname{diam}(J))^{m+1} /(1-\Phi(m+1))<+\infty$. This implies $\sum_{\sigma \in \mathbb{N}^{n}} l_{\sigma}^{m+1} \rightarrow 0$ a.s. So, $\sup _{\sigma \in \mathbf{N}^{n}} l_{\sigma}^{m+1} \rightarrow 0$ a.s. Therefore,

$$
\text { if } \Phi(0)>1, \quad \sup _{\sigma \in \mathrm{N}^{n}} l_{\sigma} \rightarrow 0 \quad \text { a.s. }
$$

THEOREM 1.3. Suppose $\Phi(0)>1$. Then almost surely the Hausdorff dimension of $K$ is $\leq \alpha$.

PROOF. Since $\Phi(\alpha) \leq 1$, by (1.8), $\left\{S_{\alpha, n}\right\}_{n=1}^{\infty}$ is a positive supermartingale and thus converges to some real random variable $X$. For each $\sigma \in \mathbf{N}^{*}$, there exists a random $m$-sphere $M_{\sigma}$ so that $J_{\sigma} \subseteq M_{\sigma}$ and the radius of $M_{\sigma}$ is $l_{\sigma}$. According to (1.14), $\sup _{\sigma \in \mathrm{N}^{n}} l_{\sigma} \rightarrow 0$ as $n \rightarrow \infty$. So,

$$
\alpha-m(K) \leq \lim _{n \rightarrow \infty} \sum_{\sigma \in \mathbf{N}^{n}}\left(\operatorname{diam}\left(M_{\sigma}\right)\right)^{\alpha} \leq 2^{\alpha} X<\infty
$$

where $\alpha-m$ is the $\alpha$-dimension Hausdorff measure. Q.E.D.

REMARK. If $\Phi(\alpha)=1$, then $\left\{S_{\alpha, n}\right\}_{n=1}^{\infty}$ is a martingale.

We will now begin consideration towards showing the Hausdorff dimension of $K$ is almost surely $\geq \alpha$. First, some involved calculations concerning moments seem necessary.

\section{The moments of $X$.}

THEOREM 2.1. Suppose $\Phi(0)>1, k_{0} \in \mathbf{N}$ and $E\left(\left(\sum_{n=1}^{\infty} T_{n}^{\alpha}\right)^{k_{0}}\right)<\infty$. Then $\left\{\left(S_{\alpha, n}, \exists_{n}\right)\right\}_{n=1}^{\infty}$ is $L^{p}$-bounded for all $p \in\left[1, k_{0}\right]$. Consequently, $X=\lim _{n} S_{\alpha, n}$ has a finite moment of order $k_{0}$.

It is sufficient to establish the following lemma. 
LEMMA 2.2. For every $k \in\left[1, k_{0}\right],\left\{E\left(S_{\alpha, n}^{k}\right)\right\}_{n=1}^{\infty} \in l^{\infty}$ and for any $\gamma>\alpha$ and any $z \in(0,1],\left\{\left[E\left(S_{\gamma, n}^{k}\right)\right]^{z}\right\}_{n=1}^{\infty} \in l^{1}$.

ProOF. For each $p, n \in \mathbf{N}$, let $S_{p ; \gamma, n}$ denote $\sum_{\sigma \in\{1, \ldots, p\}^{n}} l_{\sigma}^{\gamma}$. For any $k \in(0, \infty)$, $E\left(S_{p ; \gamma, n}^{k}\right) \leq(\operatorname{diam}(J))^{\gamma k} p^{n k}$. Consider

$$
E\left(S_{p ; \gamma, n+1}^{k} \mid \mathcal{F}_{n}\right)=E\left(\left(\sum_{\sigma \in\{1, \ldots, p\}^{n}} l_{\sigma}^{\gamma}\left(\sum_{j=1}^{p} T_{\sigma * j}^{\gamma}\right)\right)^{k} \mid \mathcal{F}_{n}\right) .
$$

Thus, if $k$ is also an integer,

$$
E\left(S_{p ; \gamma, n+1}^{k} \mid \mathcal{F}_{n}\right)=\sum_{f:\{1, \ldots, k\} \rightarrow \Delta} \prod_{i=1}^{k} x_{f(i)} \prod_{j=1}^{k} m_{p ; \gamma, j}^{r_{j}(f)},
$$

where $\Delta=\{1, \ldots, p\}^{n}, x_{\sigma}=l_{\sigma}^{\gamma}$ for each $\sigma \in \Delta, m_{p ; \gamma, j}=E\left[\left(\sum_{i=1}^{p} T_{i}^{\gamma}\right)^{j}\right]$ and $r_{j}(f)=\#\{A \in \operatorname{ker}(f) \mid \# A=j\}$ with $\operatorname{ker}(f)=\left\{f^{-1}(i) \mid i \in \Delta\right\}$. Notice that $\prod_{j=1}^{k} m_{p ; \gamma, j}^{r_{j}(f)}$ depends only on $\operatorname{ker}(f)$, the partition of $\{1, \ldots, k\}$ induced by $f$. So, for each partition $\pi$, let $m_{p ; \gamma, \pi}$ denote this number. Thus,

$$
E\left(S_{p ; \gamma, n+1}^{k} \mid \mathcal{F}_{n}\right)=\sum_{\pi \in P} m_{p ; \gamma, \pi} k_{\pi}
$$

where $P$ is the set of all partitions and $k_{\pi}=\sum_{\operatorname{ker}(f)=\pi} \prod_{i=1}^{k} x_{f(i)}$. Our notation is consistent with that given by Doubilet [6]. So, according to $[6$, Theorem 2, equation 13], we have

$$
E\left(S_{p ; \gamma, n+1}^{k} \mid \Im_{n}\right)=\sum_{\pi \in P} m_{p ; \gamma, \pi} \sum_{\sigma \geq \pi} \mu(\pi, \sigma) s_{\sigma} ;
$$

interchanging order of summation,

$$
=\sum_{\sigma \in P} s_{\sigma} \sum_{\pi \leq \sigma} \mu(\pi, \sigma) m_{p ; \gamma, \pi},
$$

where the Möbius function $\mu$, the order $\leq$ and the $s_{\sigma}$ 's are as given in [6].

In our particular case,

$$
s_{\sigma}=\prod_{j=1}^{k} S_{p ; j \gamma, n}^{\hat{\sigma}(j)}
$$

where $\hat{\sigma}(j)=\#\{A \in \sigma \mid \#(A)=j\}$. Now, set $B=\left\{\eta:\{1, \ldots, k\} \rightarrow Z_{+} \mid \sum_{j=1}^{k} j \eta(j)\right.$ $=k\}$, and

$$
c_{p ; \gamma, \eta}=\sum_{\substack{\sigma \in P \\ \hat{\sigma}=\eta}} \sum_{\pi \leq \sigma} \mu(\pi, \sigma) m_{p ; \gamma, \pi}
$$

We have

$$
E\left(S_{p ; \gamma, n+1}^{k} \mid \xi_{n}\right)=\sum_{\eta \in B} c_{p ; \gamma, \eta} \prod_{j=1}^{k} S_{p ; j \gamma, n}^{\eta(j)}
$$


Taking expectations, and using the triangle inequality we find

$$
E\left(S_{p ; \gamma, n+1}^{k}\right) \leq \sum_{\eta \in B}\left|c_{p ; \gamma, \eta}\right| E\left(\prod_{j=1}^{k} S_{p ; j \gamma, n}^{\eta(j)}\right)
$$

Let $m_{\gamma, j}=E\left(\left(\sum_{i=1}^{\infty} T_{i}^{\gamma}\right)^{j}\right)$. If $\gamma \geq \alpha$ and $j \in\left[0, k_{0}\right]$, then $m_{\gamma, j}<\infty$ and $m_{p ; \gamma, j} \rightarrow$ $m_{\gamma, j}$ as $p \rightarrow \infty$ for $j=1, \ldots, k$. Thus, $c_{p ; \gamma, \eta} \rightarrow c_{\gamma, \eta}$ (where $c_{\gamma, \eta}$ is defined similarly to $\left.c_{p ; \gamma, \eta}\right)$. But, $S_{p ; \gamma, q}^{j}$ converges upward to $S_{\gamma, q}^{j}$ as $p \rightarrow \infty$. Taking limits in (2.9) as $p \rightarrow \infty$, we find

$$
E\left(S_{\gamma, n+1}^{k}\right) \leq \sum_{\eta \in B}\left|c_{\gamma, \eta}\right| E\left[\prod_{j=1}^{k} S_{j \gamma, n}^{\eta(j)}\right]
$$

Let $\xi$ denote the unique element of $B$ such that $\xi(1)=k$. We calculate $c_{\gamma, \xi}$. Note there is only one $\sigma \in P$ such that $\hat{\sigma}=\xi$, namely the discrete partition. Also, if $\pi \leq \sigma$, then $\pi=\sigma$. So, according to $(2.7) c_{\gamma, \xi}=\mu(\sigma, \sigma) m_{\gamma, \sigma}=(\Phi(\gamma))^{k}$. We can rewrite (2.10) as

$$
E\left(S_{\gamma, n+1}^{k}\right) \leq \Phi(\gamma)^{k} E\left(S_{\gamma, n}^{k}\right)+\sum_{\substack{\eta \in B \\ \eta \neq \xi}}\left|c_{\gamma, \eta}\right| E\left[\prod_{j=1}^{k} S_{j \gamma, n}^{\eta(j)}\right]
$$

From this we derive by backwards recursion,

$$
E\left(S_{\gamma, n+1}^{k}\right) \leq \Phi(\gamma)^{k(n+1)} l_{0}^{\gamma k}+\sum_{\substack{\eta \in B \\ \eta \neq \xi}}\left|c_{\gamma, \eta}\right| \sum_{t=0}^{n} \Phi(\gamma)^{k(n-t)} E\left[\prod_{j=1}^{k} S_{j \gamma, t}^{\eta(j)}\right]
$$

Easily, $E\left(S_{\gamma, n}^{1}\right)=\Phi(\gamma)^{n} l_{0}^{\gamma}$ implies Lemma 2.2 for $k=1$ since $\Phi(\alpha) \leq 1$ and $\Phi(\gamma) \in(0,1)$ if $\gamma>\alpha$. As another special case, let $k=2$. Then $B$ has only two elements $(2,0)$ and $(0,1)$. Thus,

$$
E\left(S_{\gamma, n+1}^{2}\right) \leq l_{0}^{2 \gamma}\left[\Phi(\gamma)^{2(n+1)}+\left|c_{\gamma,(0,1)}\right| \sum_{t=0}^{n} \Phi(\gamma)^{2(n-t)} \Phi(2 \gamma)^{t}\right]
$$

In particular,

$$
\begin{aligned}
E\left(S_{\alpha, n+1}^{2}\right) & \leq l_{0}^{2 \alpha}\left[1+\left|c_{\alpha,(0,1)}\right| \sum_{t=0}^{n} \Phi(2 \alpha)^{t}\right] \\
& \leq l_{0}^{2 \alpha}\left[1+\frac{\left|c_{\alpha,(0,1)}\right|}{1-\Phi(2 \alpha)}\right]<\infty,
\end{aligned}
$$

since $\Phi(2 \alpha)<1$ (because $\Phi(0)>1$ ). Actually, from (2.7) we have

$$
c_{\alpha,(0,1)}=\operatorname{Var}\left(\sum_{n=1}^{\infty} T_{n}^{\alpha}\right)
$$


If $\gamma>\alpha$, then $\Phi(\gamma) \in(0,1)$ and $\Phi(2 \gamma) \in(0,1)$. Using the fact if $a, b>0$, then $(a+b)^{z} \leq a^{z}+b^{z}$ for any $z \in[0,1]$, we find

(2.15)

$$
\begin{aligned}
\sum_{n=0}^{\infty}\left[E\left(S_{\gamma, n}^{2}\right)\right]^{z} & \leq \sum_{n=0}^{\infty} \Phi(\gamma)^{2 z n} l_{0}^{2 z \gamma}+\left|c_{\gamma,(0,1)}\right|^{z} \sum_{n=0}^{\infty} \sum_{t=0}^{n} \Phi(\gamma)^{2 z(n-t)} \Phi(2 \gamma)^{z t} l_{0}^{2 z \gamma} \\
& \leq l_{0}^{2 z \gamma}\left[\frac{1}{1-\Phi(\gamma)^{2 z}}+\left|c_{\gamma,(0,1)}\right|^{z} \frac{1}{1-\Phi(\gamma)^{2 z}} \cdot \frac{1}{1-\Phi(2 \gamma)^{z}}\right]<\infty
\end{aligned}
$$

If $1<k<2$, and $z \in(0,1]$, then $\left[E\left(S_{\gamma, n}^{k}\right)\right]^{z} \leq\left[E\left(S_{\gamma, n}\right)\right]^{z}+\left[E\left(S_{\gamma, n}^{2}\right)\right]^{z}$. Thus Lemma 2.2 is true for $k \in[1,2]$.

Suppose Lemma 2.2 is true for $\tilde{k} \in[1, k-1]$, where $k \leq k_{0}$ and $k$ is an integer. If $\eta \in B$ with $\eta \neq \xi$, then $\eta(1) \leq k-2$ and $\sum_{j=1}^{k} \eta(j) \leq k-1$. Thus there are nonnegative reals $r_{1}, \ldots, r_{k}$, depending on $\eta$, so that $\eta(j) / k-1 \leq r_{j} \leq 1$ and $\sum_{j=1}^{k} r_{j}=1$. Let $p(\eta, j)=1 / r_{j}$. Then for each $\eta \in B$ with $\eta \neq \xi$, $\sum_{j=1}^{k} 1 / p(\eta, j)=1, p(\eta, j) \eta(j) \leq k-1$, and if $\eta(j) \neq 0$, then $\eta(j) p(\eta, j) \geq 1$, i.e., $p(\eta, j) \eta(j) \in[1, k-1]$ if $\eta(j) \neq 0$. By Hölder's inequality,

$$
E\left(\prod_{j=1}^{k} S_{j \gamma, n}^{\eta(j)}\right) \leq \prod_{j=1}^{k}\left\|S_{j \gamma, n}^{\eta(j)}\right\|_{p(\eta, j)}
$$

Substituting into (2.12), we find for $z \in(0,1]$

$$
\left[E\left(S_{\gamma, n}^{k}\right)\right]^{z} \leq \Phi(\gamma)^{z k n} l_{0}^{\gamma z k}+\sum_{\substack{\eta \in B \\ \eta \neq \xi}}\left|c_{\gamma, \eta}\right|^{z} \sum_{t=0}^{n-1} \Phi(\gamma)^{z k(n-1-t)} \prod_{j=1}^{k}\left\|S_{j \gamma, t}^{\eta(j)}\right\|_{p(\eta, j)}^{z}
$$

Let $W_{\eta, j}=\left\{\left\|S_{j \gamma, t}^{\eta(j)}\right\|_{p(\eta, j)}^{z}\right\}_{t=0}^{\infty}$. If $\eta(j)=0$, then obviously $W_{\eta, j} \in l^{\infty}$. If $\eta(j)>0$, then $W_{\eta, j} \in l^{\infty}$ by the induction hypothesis and $\eta(j) p(\eta, j) \in[1, k-1]$. But, since $\eta \neq \xi$, there is some $j \geq 2$ so that $\eta(j) \geq 1$ which implies $W_{\eta, j} \in l^{1}$ since $j \gamma>\alpha$ if $\gamma \geq \alpha$ and $j \geq 2$. Thus if $\gamma \geq \alpha$, then

$$
\left\{\prod_{j=1}^{k}\left\|S_{j \gamma, t}^{\eta(j)}\right\|_{p(\eta, j)}^{z}\right\}_{t=0}^{\infty} \in l^{1}
$$

Obviously, using $\Phi(\alpha) \leq 1$,

$$
\sup _{n}\left(\left[E\left(S_{\alpha, n}^{k}\right)\right]^{z}\right) \leq l_{0}^{\alpha z k}+\sum_{\substack{\eta \in B \\ \eta \neq \xi}}\left|c_{\alpha, \eta}\right|^{z}\left(\sum_{t=0}^{z} \prod_{j=1}^{k}\left\|S_{j \alpha, t}^{\eta(j)}\right\|_{p(\eta, j)}^{z}\right)<\infty .
$$

If $\gamma>\alpha$, then $\Phi(\gamma) \in(0,1)$. Using this fact, summing both sides of $(2.17)$, making a change of variables and an obvious approximation, we obtain

$$
\sum_{n=0}^{\infty}\left[E\left(S_{\gamma, n}^{k}\right)\right]^{z} \leq \frac{l_{0}^{\gamma z k}}{1-\Phi(\gamma)^{z k}}+\sum_{\substack{\eta \in B \\ \eta \neq \xi}}\left|c_{\gamma, \eta}\right|^{z} \frac{\sum_{t=0}^{\infty} \prod_{j=1}^{k}\left\|S_{j \gamma, t}^{\eta(j)}\right\|_{p(\eta, j)}^{z}}{1-\Phi(\gamma)^{z k}}<\infty
$$

Since for any $r \in[k-1, k]$ and $z \in(0,1]$,

$$
\left[E\left(S_{\gamma, n}^{r}\right)\right]^{z} \leq\left[E\left(S_{\gamma, n}^{k-1}\right)\right]^{z}+\left[E\left(S_{\gamma, n}^{k}\right)\right]^{z}
$$

we have extended Lemma 2.2 to $[1, k]$. This completes the induction. Q.E.D. 
3. The random construction measure. We shall now define, for almost all $\omega$, a bounded countably additive measure $\nu_{\omega}$ on the Borel sets of $\mathbf{R}^{m}$ satisfying:

(1) $\nu_{\omega}$ has total mass $X(\omega)$,

(2) $\nu_{\omega}(K(\omega))=X(\omega)$.

We shall call $\nu$ the random measure of the construction $\mathbf{J}$. Throughout this section $\alpha$ is the number defined in Theorem 1.1.

First, we define the random functional $F$ on $C_{c}\left(\mathbf{R}^{m}\right)=\left\{f \in C\left(\mathbf{R}^{m}\right) \mid f\right.$ has compact support\}: for $f \in C_{c}\left(\mathbf{R}^{m}\right)$,

$$
F(f)=\lim _{n \rightarrow \infty} \sum_{\sigma \in \mathbf{N}^{n}} f\left(s_{\sigma}\right) l_{\sigma}^{\alpha}
$$

where $s_{\sigma} \in J_{\sigma}$ when $J_{\sigma} \neq \varnothing$.

THEOREM 3.1. Suppose $\Phi(0)>1$. For almost all $\omega$ and for all $f \in C_{c}\left(\mathbf{R}^{m}\right)$, $F_{\omega}(f)$ is well defined and is a positive linear functional of norm $X(\omega)$.

Proof. For each $\sigma \in \mathbf{N}^{*}$, we define a random variable $X_{\sigma}$ by

$$
X_{\sigma}=\lim _{n \rightarrow \infty} S_{\sigma ; n}
$$

where

$$
S_{\sigma ; n} \equiv \sum_{\eta \in \mathbf{N}^{n}} \prod_{t=1}^{n} T_{\sigma *[\eta \mid t]}^{\alpha} .
$$

For each $\sigma \in \mathbf{N}^{*}, X_{\sigma}$ exists almost surely and has the same distribution as $X /(\operatorname{diam}(J))^{\alpha}$. Further for each $n \in \mathbf{N},\left\{X_{\sigma}: \sigma \in \mathbf{N}^{n}\right\}$ is an independnet family and, as a family, independent of $\mathcal{F}_{n}$. We note

$$
X=\sum_{\sigma \in \mathbf{N}^{n}} l_{\sigma}^{\alpha} X_{\sigma}
$$

The set $\Omega^{\prime}=\left\{\omega \in \Omega \mid \forall \sigma \in \mathbf{N}^{*}, X_{\sigma}(\omega)\right.$ exists and $\left.\lim _{n \rightarrow \infty} \sup _{\gamma \in \mathbf{N}^{n}} l_{\gamma}(\omega)=0\right\}$ has probability one. Suppose $f \in C_{c}\left(\mathbf{R}^{m}\right)$. For convenience, if $p, q \in \mathbf{N}$, then let $\varepsilon_{p, q}(\omega)$ denote

$$
\left|\sum_{\sigma \in \mathbf{N}^{p}} f\left(s_{\sigma}(\omega)\right) l_{\sigma}^{\alpha}(\omega)-\sum_{\sigma \in \mathbf{N}^{q}} f\left(s_{\sigma}(\omega)\right) l_{\sigma}^{\alpha}(\omega)\right| .
$$

Temporarily fix $k \in \mathbf{N}$ and suppose $p, q \geq k$. Then

$$
\begin{aligned}
\varepsilon_{p, q} & =\left|\sum_{\sigma \in \mathbf{N}^{k}} l_{\sigma}^{\alpha}\left(\sum_{\eta \in \mathbf{N}^{p-k}} f\left(s_{\sigma * \eta}\right) \prod_{t=1}^{p-k} T_{\sigma *[\eta \mid t]}^{\alpha}-\sum_{\eta \in \mathbf{N}^{q-k}} f\left(s_{\sigma * \eta}\right) \prod_{t=1}^{q-k} T_{\sigma *[\eta \mid t]}^{\alpha}\right)\right| \\
\leq & \sum_{\sigma \in \mathbf{N}^{k}} l_{\sigma}^{\alpha}\left[\sup _{\eta \in \mathbf{N}^{p-k}}\left|f\left(s_{\sigma * \eta}\right)-f\left(s_{\sigma}\right)\right| S_{\sigma ; p-k}+\left|f\left(s_{\sigma}\right)\right|\left|S_{\sigma ; p-k}-S_{\sigma ; q-k}\right|\right. \\
& \left.+\sup _{\eta \in \mathbf{N}^{q-k}}\left|f\left(s_{\sigma * \eta}\right)-f\left(s_{\sigma}\right)\right| S_{\sigma ; q-k}\right] \\
\leq & \sum_{\sigma \in \mathbf{N}^{k}} l_{\sigma}^{\alpha}\left[\operatorname{diam}\left(f\left(J_{\sigma}\right)\right)\left(S_{\sigma ; p-k}+S_{\sigma ; q-k}\right)+\|f\|_{\infty}\left|S_{\sigma ; p-k}-S_{\sigma ; q-k}\right|\right] .
\end{aligned}
$$


Thus,

$$
\begin{aligned}
\varlimsup_{p, q \rightarrow \infty} \varepsilon_{p, q}(\omega) & \leq 2 \sum_{\sigma \in \mathbf{N}^{k}} l_{\sigma}^{\alpha}(\omega) \operatorname{diam}\left(f\left(J_{\sigma}(\omega)\right)\right) X_{\sigma}(\omega) \\
& \leq 2 \sup _{\sigma \in \mathbf{N}^{k}} \operatorname{diam}\left(f\left(J_{\sigma}(\omega)\right)\right) X(\omega)
\end{aligned}
$$

if $\omega \in \Omega^{\prime}$. But, if $\omega \in \Omega^{\prime}, \sup _{\sigma \in \mathbf{N}^{k}} \operatorname{diam}\left(f\left(J_{\sigma}(\omega)\right)\right) \rightarrow 0$ as $k \rightarrow \infty$. Thus,

$$
\varlimsup_{p, q \rightarrow \infty} \varepsilon_{p, q}(\omega)=0 \quad \text { if } \omega \in \Omega^{\prime} .
$$

Obviously, $F_{\omega}$ is linear positive and for any $f \in C_{c}\left(\mathbf{R}^{m}\right)$ so that $J \subseteq f^{-1}(1)$, we have $F_{\omega}(f)=\lim _{n \rightarrow \infty} \sum_{\sigma \in \mathbb{N}^{n}} l_{\sigma}^{\alpha}(\omega)=K(\omega)$. Thus, $\left\|F_{\omega}\right\|=X(\omega)$. We let $\nu_{\omega}$ denote the Borel measure on $\mathbf{R}^{m}$ so that $F_{\omega}(f)=\int_{\mathbf{R}^{m}} f(x) d \nu_{\omega}(x)$.

THEOREM 3.2. Suppose $\Phi(0)>1$. If $A$ is a compact subset of $\mathbf{R}^{m}$, then

$$
\nu(A)=\lim _{n \rightarrow \infty} \sum_{\substack{\sigma \in \mathbb{N}^{n} \\ J_{\sigma} \cap A \neq \varnothing}} l_{\sigma}^{\alpha} X_{\sigma} \quad \text { a.s. }
$$

In fact,

$$
\sum_{\substack{\sigma \in \mathbf{N}^{n} \\ J_{\sigma} \cap A \neq \varnothing}} l_{\sigma}^{\alpha} X_{\sigma} \downarrow \nu(A) \quad \text { as } n \rightarrow \infty \text { a.s. }
$$

Proof. Fix $\omega \in \Omega^{\prime}$ and $k \in \mathbf{N}$. Let $\varepsilon>0$. Since $\sum_{\sigma \in \mathbf{N}^{k}} l_{\sigma}^{\alpha}(\omega) X_{\sigma}(\omega)$ is finite, there is a finite set $M \subseteq \mathbf{N}^{k}$ such that $\sum_{\sigma \in \mathbf{N}^{k} \backslash M} l_{\sigma}^{\alpha}(\omega) X_{\alpha}(\omega)<\varepsilon$. Let $f$ be a continuous map of $\mathbf{R}^{m}$ into $[0,1]$ such that $f^{-1}(1)=A$ and $J_{\sigma} \subseteq f^{-1}(0)$ if $\sigma \in M$ and $J_{\sigma} \cap A=\varnothing$. Now

$$
\begin{aligned}
\nu_{\omega}(A) \leq & \int_{\mathbf{R}^{m}} f d \nu_{\omega}=\lim _{n \rightarrow \infty} \sum_{\sigma \in \mathbf{N}^{n}} f\left(s_{\sigma}(\omega)\right) l_{\sigma}^{\alpha}(\omega) \\
& =\lim _{n \rightarrow \infty} \sum_{\sigma \in \mathbf{N}^{k}} \sum_{\eta \in \mathbf{N}^{n-k}} f\left(s_{\sigma * \eta}(\omega)\right) l_{\sigma * \eta}^{\alpha}(\omega) \\
\leq & \lim _{n \rightarrow \infty} \sum_{\substack{\sigma \in \mathbf{N}^{k} \\
J_{\sigma}(\omega) \cap A \neq \varnothing}} \sum_{\eta \in \mathbf{N}^{n-k}} l_{\sigma * \eta}^{\alpha}(\omega) \\
& +\lim _{n \rightarrow \infty} \sum_{\substack{\sigma \in \mathbf{N}^{k} \backslash M \\
J_{\sigma}(\omega) \cap A=\varnothing}} \sum_{\eta \in \mathbf{N}^{n-k}} l_{\sigma * \eta}^{\alpha}(\omega) .
\end{aligned}
$$

Note

$$
\lim _{n \rightarrow \infty} \sum_{\sigma \in D \subseteq \mathbf{N}^{k}} \sum_{\eta \in \mathbf{N}^{n-k}} l_{\sigma * \eta}^{\alpha}(\omega)=\sum_{\sigma \in D} l_{\sigma}(\omega) X_{\sigma}(\omega) .
$$

So,

$$
\nu_{\omega}(A) \leq \sum_{\substack{\sigma \in \mathbf{N}^{k} \\ J_{\sigma}(\omega) \cap A \neq \varnothing}} l_{\sigma}(\omega) X_{\sigma}(\omega)+\varepsilon
$$


Or,

$$
\nu_{\omega}(A) \leq \sum_{\substack{\sigma \in \mathbf{N}^{k} \\ J_{\sigma}(\omega) \cap A \neq \varnothing}} l_{\sigma}(\omega) X_{\sigma}(\omega)
$$

Since, for any $\sigma \in \mathbf{N}^{*}, l_{\sigma}^{\alpha} X_{\sigma}=\sum_{n=1}^{\infty} l_{\sigma * n}^{\alpha} X_{\sigma * n}$, we have

$$
\sum_{\substack{\sigma \in \mathbf{N}^{k} \\ J_{\sigma}(\omega) \cap A \neq \varnothing}} l_{\sigma}^{\alpha}(\omega) X_{\sigma}(\omega) \geq \sum_{\substack{\sigma \in \mathbf{N}^{k+1} \\ J_{\sigma}(\omega) \cap A \neq \varnothing}} l_{\sigma}^{\alpha}(\omega) X_{\sigma}(\omega) .
$$

Let $\mu_{\omega}(A)=\lim _{n} \sum_{\sigma \in \mathbf{N}^{n} ; A \cap J_{\sigma}(\omega) \neq \varnothing} l_{\sigma}^{\alpha}(\omega) X_{\sigma}(\omega)$. Suppose $B$ is a compact subset of $\mathbf{R}^{m}$ disjoint from $A$; then there is a $k \in \mathbf{N}$ so that if $\sigma \in \mathbf{N}^{k}$, then $J_{\sigma}$ meets at most one of $A$ and $B$. Thus, $\mu_{\omega}(A)+\mu_{\omega}(B) \leq X(\omega)$. Further,

$$
\nu_{\omega}(A)+\nu_{\omega}(B) \leq \mu_{\omega}(A)+\nu_{\omega}(B) \leq \mu_{\omega}(A)+\mu_{\omega}(B) \leq X(\omega) .
$$

Find compact sets $B_{1} \subseteq B_{2} \subseteq B_{3} \subseteq \cdots \subseteq A^{c}$ so that $\nu\left(B_{n}\right) \uparrow \nu\left(A^{c}\right)$. We see

$$
X(\omega)=\nu_{\omega}(A)+\nu_{\omega}\left(A^{c}\right) \leq \mu_{\omega}(A)+\nu_{\omega}\left(A^{c}\right) \leq X(\omega)
$$

i.e., $\mu_{\omega}(A)=\nu_{\omega}(A)$. Q.E.D.

THEOREM 3.3. Suppose $\Phi(0)>1$. Almost surely $\nu(K)=X$.

PROOF. Since $K=\bigcap_{n=1}^{\infty} \bigcup_{\sigma \in \mathbb{N}^{n}} J_{\sigma}$ is a nested intersection,

$$
\nu(K)=\lim _{n \rightarrow \infty} \nu\left(\bigcup_{\sigma \in \mathbf{N}^{n}} J_{\sigma}\right) \text {. }
$$

Temporarily fix $n$ and let $\Delta_{1} \subseteq \Delta_{2} \subseteq \Delta_{3} \subseteq \cdots$ be an increasing sequence of finite subsets of $\mathbf{N}^{n}$ such that $\bigcup \Delta_{j}=\mathbf{N}^{n}$. According to Theorem 3.2, we have, for each $i$

$$
\begin{aligned}
\nu\left(\bigcup_{\sigma \in \Delta_{i}} J_{\sigma}\right) & =\lim _{k \rightarrow \infty} \sum_{\substack{\eta \in \mathbf{N}^{k} \\
J_{\eta} \cap J_{\sigma} \neq \varnothing \\
\text { for some } \sigma \in \Delta_{i}}} l_{\eta}^{\alpha} X_{\eta} \\
& \geq \varlimsup_{k \rightarrow \infty} \sum_{\sigma \in \Delta_{i}} \sum_{\eta \in \mathbf{N}^{k}} l_{\sigma * \eta}^{\alpha} X_{\sigma * \eta}=\sum_{\sigma \in \Delta_{j}} l_{\sigma}^{\alpha} X_{\sigma} .
\end{aligned}
$$

Thus,

$$
X \geq \nu\left(\bigcup_{\sigma \in \mathbb{N}^{n}} J_{\sigma}\right) \geq \sum_{\sigma \in \Delta_{j}} l_{\sigma}^{\alpha} X_{\sigma} \underset{j \rightarrow \infty}{\rightarrow} \sum_{\sigma \in \mathbb{N}^{n}} l_{\sigma}^{\alpha} X_{\sigma}=X . \quad \text { Q.E.D. }
$$

THEOREM 3.4. If $E(X)>0$, then $P(\nu(K)>0 \mid K \neq \varnothing)=1$.

Proof. By hypothesis, $E(X)>0$ and so $P(X>0)=\delta>0$. Recall that for each $\sigma \in \mathrm{N}^{n}, X_{\sigma}$ exists a.s. and has the same distribution as $X /(\operatorname{diam} J)^{\alpha}$. Further, for each $n \in \mathbf{N},\left\{X_{\sigma} \mid \sigma \in \mathbf{N}^{n}\right\}$ is an independent family and, as a family, also independent of $\mathcal{F}_{n}$. We note again

$$
X=\sum_{\sigma \in \mathbb{N}^{n}} l_{\sigma}^{\alpha} X_{\sigma} .
$$


As in the proof of Theorem 1.2 we consider two cases:
A: $\quad P\left(\sum_{n=1}^{\infty} T_{n}^{0}<\infty\right)=1$ and
B: $\quad P\left(\sum_{n=1}^{\infty} T_{n}^{0}=\infty\right)>0$.

Case A. We will establish that for each $n$ and $q$,

$$
P\left(X>0 \text { and } S_{0, n} \geq q\right) \leq(1-\delta)^{q} P\left(S_{0, n} \geq q\right) .
$$

Since $P\left(\sum_{n=1}^{\infty} T_{n}^{0}<\infty\right)=1$, the random index set $D_{n}=\left\{\sigma \in \mathbf{N}^{n} \mid l_{\sigma}>0\right\}$ is finite. Let $F$ be a finite subset of $\mathbf{N}^{n}$. Using (3.15) we see

$$
\begin{aligned}
P\left(X=0 \text { and } F=D_{n}\right) & =P\left(X_{\sigma}=0 \text { for } \sigma \in F \text { and } F=D_{n}\right) \\
& =(1-\delta)^{\# F} P\left(D_{n}=F\right),
\end{aligned}
$$

due to independence. Now, inequality (3.16) follows by summing over finite $F$ such that $\# F \geq q$ and using $(1-\delta)^{\# F} \leq(1-\delta)^{q}$.

From (3.16), we calculate

$$
P(X>0) \geq P\left(X>0 \text { and } S_{0, n} \geq q\right) \geq\left[1-(1-\delta)^{q}\right] P\left(S_{0, n} \geq q\right) .
$$

Noting $P\left(S_{0, n} \geq q\right) \rightarrow P(S=\infty)$ as $n \rightarrow \infty$, and letting $n \rightarrow \infty$ in (3.17), and then letting $q \rightarrow \infty$, we find

$$
P(X>0) \geq P(S=\infty) .
$$

But a.s. "if $S=0$, then there is some $n \in \mathbf{N}$ so that $S_{0, n}=0$; i.e., $X=$ $\sum_{\sigma \in \mathbf{N}^{n}} l_{\sigma} X_{\sigma}=0 "$. Thus,

$$
\text { "if } X>0 \text {, then } S=\infty \text { " a.s. }
$$

Combining (3.18), (3.19), Theorems 1.2 and 1.3, we find

" $K \neq \varnothing$ if and only if $X>0$ if and only if $\nu(K)>0 "$ a.s.

Case B. Suppose $P\left(\sum_{n=1}^{\infty} T_{n}^{0}=\infty\right)>0$. As in the proof of Theorem 1.2, with probability one, "if $S=\infty$, then there is some $\sigma \in \mathbf{N}^{k}$ such that $J_{\sigma} \neq$ $\varnothing$ and $\sum_{n=1}^{\infty} T_{\sigma * n}^{0}=\infty "$. But, since $\left\{X_{\sigma * n}\right\}_{n=1}^{\infty}$ is an independent family with common distribution, the distribution of $X /(\operatorname{diam} J)^{\alpha}$, and since this family is independent of $\mathcal{F}_{|\sigma|+1}$, we have with probability one "if $J_{\eta} \neq \varnothing$ and $\sum_{n=1}^{\infty} T_{\eta * n}^{0}=$ $\infty$, then there is some $k \in \mathbf{N}$ such that $T_{\eta * k}^{0}=1$ and $X_{\eta * k}>0$, i.e., $X=$ $\sum_{\sigma \in \mathrm{N}|\eta|} l_{\sigma}^{\alpha} X_{\sigma} \geq l_{\sigma * k}^{\alpha} X_{\eta * k}=l_{\eta}^{\alpha} T_{\eta * k}^{\alpha} X_{\eta * k}>0$ ". Thus, we may conclude with the following statement: Almost surely "if $S=\infty$, then $X>0$ ". Q.E.D.

The next theorem is a probabilistic mixing of the deterministic methods of Rogers and Taylor [16] and Moran [15].

THEOREM 3.5. Suppose the construction $\mathbf{J}$ is such that there exists $\delta>0$ so that with probability one, if $T_{i}(\omega)>0$, then $T_{i}(\omega)>\delta$. If $\Phi(0)>1$, then, for almost all $\omega$, for all $\beta<\alpha$ and for all Borel $E \subseteq \mathbf{R}^{m}$, if $\beta-m(E)<\infty$, then $\nu_{\omega}(E)=0$.

PROOF. Since for any $\gamma \geq 0, \delta^{m} \cdot \sum_{i=1}^{\infty} T_{i}^{\gamma} \leq \sum_{i=1}^{\infty} T_{i}^{m} \leq 1$ a.s., we have $\sum_{i=1}^{\infty} T_{i}^{\gamma} \leq 1 / \delta^{m}$ a.s. It follows that

$$
E\left(\left(\sum_{i=1}^{\infty} T_{i}^{\gamma}\right)^{t}\right) \leq\left(1 / \delta^{m}\right)^{t}<\infty
$$


Consequently, by Theorem 2.1, $E\left(X^{t}\right)<\infty$ for all $t \in(0, \infty)$. Fix $k>0$, and $\beta<\alpha$; then for any $t>0$,

$$
P\left(l_{\sigma}^{\alpha} X_{\sigma}>k l_{\sigma}^{\beta}\right) \leq \frac{E\left(\left[l_{\sigma}^{\alpha-\beta}\right]^{t} X_{\sigma}^{t}\right)}{k^{t}}
$$

and, by independence,

$$
=\frac{E\left(l_{\sigma}^{(\alpha-\beta) t}\right) E\left(X^{t}\right)}{\operatorname{diam}(J)^{\alpha t} k^{t}}
$$

which implies

$$
\begin{aligned}
\sum_{\sigma \in \mathbf{N}^{n}} P\left(l_{\sigma}^{\alpha} X_{\sigma}>k l_{\sigma}^{\beta}\right) & \leq \frac{E\left(S_{(\alpha-\beta) t, n}\right) E\left(X^{t}\right)}{\operatorname{diam}(J)^{\alpha t} k^{t}} \\
& =\frac{\Phi((\alpha-\beta) t)^{n} E\left(X^{t}\right)}{k^{t} \operatorname{diam}(J)^{\alpha t}}
\end{aligned}
$$

Choose $t_{0}$ such that $\Phi\left((\alpha-\beta) t_{0}\right)<1$. Thus,

$$
\begin{aligned}
\sum_{n=0}^{\infty} P & \left(\exists \sigma \in \mathbf{N}^{n} \ni l_{\sigma}^{\alpha} X_{\sigma}>k l_{\sigma}^{\beta}\right) \\
& \leq \sum_{n=0}^{\infty} \frac{\Phi\left((\alpha-\beta) t_{0}\right)^{n} E\left(X^{t_{0}}\right)}{k^{t_{0}} \operatorname{diam}(J)^{\alpha t_{0}}}<\infty .
\end{aligned}
$$

Thus by the Borel-Cantelli lemma, $P\left(\exists N \ni \forall n \geq N\right.$, if $\sigma \in \mathbf{N}^{n}$, then $l_{\sigma}^{\alpha} X_{\sigma} \leq$ $\left.k l_{\sigma}^{\beta}\right)=1$. Fix $\omega$ so that $\nu_{\omega}$ is defined and for which there is $N(\omega) \in \mathbf{N}$ so that if $\sigma \in \mathbf{N}^{n}, n \geq N(\omega)$, then $l_{\sigma}^{\alpha}(\omega) X_{\sigma}(\omega) \leq k l_{\sigma}^{\beta}(\omega)$. For each $x \in K(\omega)$ and $p \in \mathbf{N}$, let $\Delta_{x, p} \equiv\left\{\sigma \in \mathbf{N}^{*} \mid x \in J_{\sigma}(\omega), l_{\sigma}(\omega)=\operatorname{diam}\left(J_{\alpha}(\omega)\right)<2^{-p}\right.$ and $\operatorname{diam}\left(J_{\sigma|| \sigma \mid-1}(\omega)\right) \geq$ $\left.2^{-p}\right\}$.

Suppose $E$ is a compact subset of $\mathbf{R}^{m}$ with $\beta-m(E)<\infty$. Suppose $L_{1}, L_{2}, \ldots$ is a cover of $E$ by closed $m$-spheres so that $\operatorname{diam}\left(L_{i}\right)<\varepsilon / 2$ for each $i$, where $\varepsilon=1 \wedge \min \left\{l_{\sigma}(\omega)>0 \mid \sigma \in \mathbf{N}^{N(\omega)}\right\}$. For each $i$, there exists a $p_{i} \in \mathbf{N}$ so that $2^{-1-p_{i}} \leq \operatorname{diam}\left(L_{i}\right)<2^{-p_{i}}$. For each $i$, consider $D_{i}=\bigcup\left\{\Delta_{x, p_{i}} \mid x \in L_{i} \cap E \cap K(\omega)\right\}$. Now, $D_{i}$ is an antichain in the lattice $\mathbf{N}^{*}$ with the natural partial order: $\sigma \leq \eta$ iff $\exists \xi \ni \sigma * \xi=\eta$. Thus, the set $\left\{J_{\sigma}(\omega): \sigma \in D_{i}\right\}$ is a nonoverlapping collection. For any $y \in L_{i}, \bigcup_{\sigma \in D_{i}} J_{\sigma} \subseteq B\left(y, 2^{1-p_{i}}\right)$. By the definition of $\Delta_{x, p_{i}}$, if $\sigma \in \Delta_{x, p_{i}}$, then $\operatorname{diam}\left(J_{\sigma}(\omega)\right) \geq \delta 2^{-p_{i}}$, implying

$$
\lambda\left(\operatorname{Int} J_{\sigma}(\omega)\right) \geq\left[\frac{\delta \cdot 2^{-p_{i}}}{\operatorname{diam}(J)}\right]^{m} \lambda(\operatorname{Int} J) .
$$

Let $N_{i}$ denote the cardinality of $D_{i}$; then

$$
\begin{aligned}
N_{i}\left[\frac{\delta \cdot 2^{-p_{i}}}{\operatorname{diam}(J)}\right]^{m} \lambda(\operatorname{int}(J)) & \leq \sum_{\sigma \in D_{i}} \lambda\left(\operatorname{Int} J_{\sigma}(\omega)\right) \\
& \leq \lambda\left(B\left(y, 2^{1-p_{i}}\right)\right)=2^{-p_{i} m} \lambda(B(0,2))
\end{aligned}
$$

i.e.,

$$
N_{i} \leq\left[\frac{\operatorname{diam}(J)}{\delta}\right]^{m} \frac{\lambda(B(0,2))}{\lambda(\operatorname{Int}(J))} \equiv M<\infty
$$


For any $\sigma \in D_{i}, l_{\sigma}^{\alpha}(\omega) X_{\sigma}(\omega) \leq k l_{\sigma}^{\beta} \leq k\left[2^{-p_{i}}\right]^{\beta} \leq 2^{\beta} k \operatorname{diam}\left(L_{i}\right)^{\beta}$, implying,

$$
\sum_{\sigma \in D_{i}} l_{\sigma}^{\alpha}(\omega) X_{\sigma}(\omega) \leq 2^{\beta} k M \operatorname{diam}\left(L_{i}\right)^{\beta} .
$$

For each $i$, let $n(i)$ denote $\max \left\{|\sigma|: \sigma \in D_{i}\right\}$. Then

$$
\nu_{\omega}\left(E \cap L_{i}\right) \leq \sum_{\substack{\sigma \in N^{n(i)} \\ E \cap L_{i} \cap J_{\sigma} \neq \varnothing}} l_{\sigma}^{\alpha}(\omega) X_{\sigma}(\omega) \leq \sum_{\sigma \in D_{i}} l_{\sigma}^{\alpha}(\omega) X_{\sigma}(\omega),
$$

by Theorem 3.2 and the fact $l_{\sigma}^{\alpha}(\omega) X_{\sigma}(\omega)=\sum_{n=1}^{\infty} l_{\sigma * n}^{\alpha}(\omega) X_{\sigma * n}(\omega)$. Combining (3.24) and (3.25) we find $\nu_{\omega}(E) \leq 2^{\beta} k M \sum_{i} \operatorname{diam}\left(L_{i}\right)^{\beta}$. Therefore, $\nu_{\omega}(E) \leq$ $2^{\beta} k M \beta-m(E) \rightarrow 0$ as $k \rightarrow 0$. Q.E.D.

THEOREM 3.6. Suppose $\Phi(0)>1$. Then $P(K$ has Hausdorff $\operatorname{dim} \geq \alpha \mid K \neq$ $\varnothing)=1$.

ProOF. For each $n \in \mathbf{N}$, we define an auxiliary construction $\mathbf{J}_{n}=\left\{J_{n ; \sigma} \mid \sigma \in\right.$ $\left.\mathbf{N}^{*}\right\}$ by

$$
J_{n ; \sigma}= \begin{cases}J_{\sigma}, & \text { if } l_{[\sigma \mid k]} \geq(1 / n) l_{[\sigma \mid k-1]} \text { for } k=1, \ldots,|\sigma|, \\ \varnothing, & \text { otherwise }\end{cases}
$$

and $T_{n ; \sigma}=1_{[1 / n, \infty)}\left(T_{\sigma}\right) \cdot T_{\sigma}$.

Now,

$$
\Phi_{n}(\beta)=E\left(\sum_{p=1}^{\infty} 1_{[1 / n, \infty)}\left(T_{\sigma}\right) T_{\sigma}^{\beta}\right) \uparrow \Phi(\beta),
$$

by the monotone convergence theorem. Thus, there is some $N_{0} \in \mathbf{N}$ so that for all $n \geq N_{0}, \Phi_{n}(0)>1$.

Let $K_{n}(\omega)=\bigcap_{p=1}^{\infty} \bigcup_{\sigma \in \mathbb{N}^{p}} J_{n ; \sigma}(\omega)$. Obviously,

$$
K_{n}(\omega) \subseteq K_{n+1}(\omega) \subseteq K(\omega)
$$

for all $n$. (For later use, note $S_{n ; \gamma, k}=\sum_{\sigma \in \mathbf{N}^{k}} l_{n ; \sigma}^{\gamma}$.)

For each $n$, let $\gamma_{n}=P\left(K_{n}\right.$ is empty $)$ and let $\gamma_{0}=P(K$ is empty $)$. For each $n, p \in \mathrm{N}$, let $C_{n ; p}$ denote $P\left(\sum_{q=1}^{\infty} T_{n ; q}^{0}=p\right)$, and $C_{0 ; p}$ denote $P\left(\sum_{q=1}^{\infty} T_{q}^{0}=p\right)$. By a well-known formula (see $[\mathbf{1}]$ ):

$$
\gamma_{p}=\sum_{q=0}^{\infty} C_{p ; q} \gamma_{p}^{q}
$$

(This formula is correct even if $P\left(\sum T_{n}^{0}=\infty\right)>0$.)

Set $\psi_{p}(x)=-x+\sum_{q=0}^{\infty} C_{p ; q} x^{q}$. Clearly, $\psi_{p}$ is defined on $[0,1]$ for each $p$. Also, for $p \geq N_{0}$ or $p=0, \gamma_{p}$ is the unique root of $\psi_{p}$ in $[0,1)$ (see $[1, p .4]$ ). Moreover, $\psi_{p}$ is strictly convex for $p \geq N_{0}$ and $\psi_{0}$ is either strictly convex or linear with negative slope. By (3.27), $\gamma_{p} \geq \gamma_{p+1} \geq \gamma_{0}$ for $p \in \mathbf{N}$. Thus, $\gamma_{\infty}=\lim _{p \rightarrow \infty} \gamma_{p}$ exists and $\gamma_{\infty} \geq \gamma_{0}$. Now $C_{p ; q} \rightarrow C_{0 ; q}$ as $p \rightarrow \infty$ if $q<\infty$. Easily, $\left\{\psi_{n}\right\}_{n=1}^{\infty}$ converges uniformly to $\psi_{0}$ on $\left[0, \gamma_{N_{0}}\right]$. Thus, $0=\psi_{n}\left(\gamma_{n}\right) \rightarrow \psi_{0}\left(\gamma_{\infty}\right)$, i.e., $\psi_{0}\left(\gamma_{\infty}\right)=0$. This implies $\gamma_{\infty}=\gamma_{0}$ or

$$
\lim _{n \rightarrow \infty} P\left(K_{n}(\omega)=\varnothing\right)=P(K(\omega)=\varnothing)
$$


Now (3.29) together with (3.27) imply:

For almost all $\omega, " K(\omega) \neq \varnothing$ iff for some $n \geq N, K_{n}(\omega) \neq \varnothing "$.

For each $n$, let $\alpha_{n}$ be the " $\alpha$ " for the construction $\mathbf{J}_{n}$. Note that since $T_{n ; i} \leq 1$, for all $i$ and $\beta>0$,

$$
\sum_{k=1}^{\infty} T_{n ; k}^{\beta} \leq \sum_{k=1}^{\infty} T_{n ; k}^{0}
$$

Since $T_{n ; k} \notin(0,1 / n)$, we have according to (1.5)

$$
(1 / n)^{m} \sum_{k=1}^{\infty} T_{n ; k}^{0} \leq \sum_{k=1}^{\infty} T_{n ; k}^{m} \leq 1 .
$$

Thus, for each $n, \Phi_{n}(0) \leq n^{m}$. So, for all $n \geq N_{0}, \Phi_{n}$ is finite valued, continuous, strictly decreasing, and $\Phi_{n}\left(\alpha_{n}\right)=1$. Also, $\Phi_{n} \leq \Phi_{n+1} \leq \Phi$ for each $n$. Thus, $\alpha_{n} \leq \alpha_{n+1} \leq \alpha$ for all $n \geq N_{0}$. This implies $\alpha_{\infty}=\lim _{n \rightarrow \infty} \alpha_{n} \leq \alpha$. But,

$$
1=\Phi_{n}\left(\alpha_{n}\right) \geq \Phi_{n}\left(\alpha_{\infty}\right)=E\left(\sum_{p=1}^{\infty} T_{p}^{\alpha_{\infty}} 1_{[1 / n, \infty)}\left(T_{p}\right)\right) \uparrow \Phi\left(\alpha_{\infty}\right)
$$

as $n \rightarrow \infty$. Therefore, $1 \geq \Phi\left(\alpha_{\infty}\right)$ which implies $\alpha_{\infty} \geq \alpha$, i.e., $\alpha=\alpha_{\infty}$.

We derive from (3.31) and (3.32)

$$
E\left(\left(\sum_{k=1}^{\infty} T_{n ; k}^{\alpha_{n}}\right)^{2}\right) \leq n^{2 m}
$$

Thus, by Theorem 2.1, $\left\{S_{n ; \alpha_{n}, k}\right\}_{k=1}^{\infty}$ is an $L_{2}$-bounded martingale. This implies $E\left(X_{n}\right)=E\left(S_{n ; \alpha_{n}, 0}\right)=(\lambda(J))^{\alpha_{n}}>0$.

For each $n \geq N_{0}, \mathrm{~J}_{n}$ satisfies the hypothesis of Theorem 3.5. Suppose $\beta<\alpha$ and $K(\omega) \neq \varnothing$. Then almost surely there exists an $n \geq N_{0}$ so that $\alpha_{n}>\beta$ and $K_{n}(\omega) \neq \varnothing$. By definition of $\nu_{n ; \omega}$ and Theorem 3.4, $\nu_{n ; \omega}(K(\omega))=\nu_{n ; \omega}\left(K_{n}(\omega)\right)=$ $X_{n}(\omega)>0$. Thus, by Theorem 3.5 , almost surely $\beta-m(K(\omega))=\infty$. We can conclude that if $\beta<\alpha$, then almost surely $\beta-m(K(\omega))=\infty$ if $K(\omega) \neq \varnothing$. Q.E.D.

In what has preceded, we have required of our construction only a "stochastic ratio self-similarity". In order to ask a question of interest, we will now introduce a version of a construction being "stochastically geometrically self-similar".

We let $G$ denote the set of geometric similarity maps with domain $J$. In other words,

$G=\left\{f: J \rightarrow \mathbf{R}^{n} \mid \exists \lambda \in(0, \infty)\right.$ so that for all $\left.x, y \in J,\|f(x)-f(y)\|=\lambda\|x-y\|\right\}$,

where $\|\cdot\|$ is the Euclidean norm in $\mathbf{R}^{m}$. Also, let $J_{p}$ be the $\sigma$-algebra of subsets of $\Omega$ generated by $\left\{J_{\sigma}|| \sigma \mid \leq p\right\}$.

Note 3.7. Suppose $\sigma \in \mathbf{N}^{p}, P\left(J_{\sigma} \neq \varnothing\right)>0$ and $F_{\sigma}: \Omega \times J \rightarrow \mathbf{R}^{m}$ satisfies:

(1) $F_{\sigma}$ is $J_{p} \times B(J)$-measurable.

(2) $F_{\sigma}(\omega, J)=J_{\sigma}(\omega)$, if $J_{\sigma}(\omega) \neq \varnothing$ for almost all $\omega$.

(3) $F_{\sigma} \in G$ a.s.

Define $\tilde{\mathbf{J}}_{\sigma}=\left\{\tilde{J}_{\sigma ; \eta} \mid \eta \in \mathbf{N}^{*}\right\}$ by $\tilde{J}_{\sigma ; \eta}(\omega)=\left[F_{\sigma}(\omega, \cdot)\right]^{-1}\left(J_{\sigma * \eta}(\omega)\right)$. Then given $J_{\sigma} \neq$ $\varnothing$, we have $\tilde{\mathbf{J}}_{\sigma}$ is a construction (in fact $\tilde{\tau}_{\sigma ; \varnothing}$ is equal in distribution to $\tau_{\varnothing}$ ). We 
say a construction $\mathbf{J}$ is stochastically geometrically self-similar if for each $\sigma \in \mathbf{N}^{*}$ with $P\left(J_{\sigma} \neq \varnothing\right)>0$, there is a $F_{\sigma}$ satisfying $(1)-(3)$ of Note 3.7 so that $\tilde{\mathbf{J}}_{\sigma}$ given $\tau_{\sigma} \neq \varnothing$ is distributed the same as $J$, i.e., if $B$ is a Borel subset of $\left[2^{J}\right]^{\mathbf{N}^{*}}$, then $P\left(\tilde{\mathbf{J}}_{\sigma} \in B \mid J_{\sigma} \neq \varnothing\right.$ and $\left.\mathcal{F}_{n}\right)=P(\mathbf{J} \in B)$. All the examples given in $\S 4$ have this property.

Question 3.8. Suppose $\mathbf{J}$ is a stochastically geometrically self-similar construction indexed by an $n$-ary $\{1, \ldots, n\}^{*}, n<\infty$, and $P\left(T_{i}>0 ; i=1, \ldots, n\right)=1$ and $P\left(J_{i} \cap J_{k} \neq \varnothing\right.$ if $\left.(i \neq j)\right)=0$. Then is it true for almost all $\omega$ that

$$
0<\alpha-m(K(\omega)) ?
$$

For the deterministic case, this was proved by Moran [15]. At least we know the following facts in this case. Let $a=E(\alpha-m(K))$. Since our construction is geometrically self-similar, for $|\sigma|=k$, we have

$$
a=E\left(\alpha-m\left(\tilde{K}_{\sigma}\right) \mid J_{k} \text { and } J_{\sigma} \neq \varnothing\right) .
$$

Also, since for these constructions $J_{\sigma} \neq \varnothing$ a.s., we have

$$
a=E\left(\alpha-m\left(\tilde{K}_{\sigma}\right) \mid J_{k}\right)
$$

We now claim

$$
\alpha-m(K)=\frac{a X}{(\operatorname{diam}(J))^{\alpha}}
$$

To see this, note

$$
\alpha-m(K)=\sum_{\sigma \in\{1, \ldots, n\}^{k}} \alpha-m\left(K \cap J_{\sigma}\right)=\sum_{\sigma \in\{1, \ldots, n\}^{k}} \alpha-m\left(K_{\sigma}\right),
$$

where $K_{\sigma}$ is the set obtained by pruning the tree to start at $\sigma$ with $J_{\sigma}$. For $\sigma \in\{1, \ldots, n\}^{k}$, let $F_{\sigma}$ be a random geometric self-similarity map,

$$
\alpha-m(K(\omega))=\frac{1}{[\operatorname{diam}(J)]^{\alpha}} \sum_{\sigma \in\{1, \ldots, n\}^{k}} l_{\sigma}^{\alpha} \alpha-m\left(F_{\sigma}(\omega, \cdot)\right)^{-1}\left(K_{\sigma}(\omega)\right) .
$$

This implies

$$
\begin{aligned}
E\left(\alpha-m(K) \mid J_{k}\right) & =\frac{1}{[\operatorname{diam}(J)]^{\alpha}} \sum_{\sigma \in\{1, \ldots, n\}^{k}} l_{\sigma}^{\alpha} E\left[\alpha-m\left(\tilde{K}_{\sigma}\right) \mid J_{k}\right] \\
& =\frac{a}{[\operatorname{diam}(J)]^{\alpha}} S_{\alpha, k} .
\end{aligned}
$$

Now, letting $k \rightarrow \infty$, we find

$$
\alpha-m(K)=\frac{a X}{[\operatorname{diam}(J)]^{\alpha}} .
$$

Using Theorem 2.1, it is easy to see $\left\{S_{\alpha, n}\right\}_{n=1}^{\infty}$ is a $L^{2}$-bounded martingale and $E(X)>0$. Thus, by Theorem 3.4 , we see $X>0$ a.s., so either $\alpha-m(K)>0$ a.s. or $\alpha-m(K)=0$ a.s. 


\section{Examples.}

EXAMPLE 4.1. Let us note that the following simple deterministic process shows that $X(\omega)$ may not yield $\alpha-m(K(\omega))$. Set $J_{\varnothing}=[0,1], J_{0}=\left[\frac{1}{8}, \frac{3}{8}\right]$ and $J_{1}=\left[\frac{5}{8}, \frac{7}{8}\right]$. Given $J_{\sigma}=[a, b]$, set

$$
J_{\sigma * 0}=[a+(b-a) / 8, a+3(b-a) / 8]
$$

and

$$
J_{\sigma * 1}=[a+5(b-a) / 8, a+7(b-a) / 8] \text {. }
$$

The Hausdorff dimension of the final Cantor set is $\alpha=\frac{1}{2}$. In this construction $X(\omega)=1$ a.s., whereas it can be shown that $\frac{1}{2}-m(K(\omega))=\sqrt{\frac{2}{3}}$.

EXAMPLE 4.2. We return to the example described in the introduction of a random Cantor subset of $[0,1]$. It follows from Theorem 1.1 that the Hausdorff dimension of such a set in this construction is the number $\alpha$ such that

$$
1=E\left(T_{1}^{\alpha}+T_{2}^{\alpha}\right)=2 \int_{0}^{1} \int_{x}^{1} x^{\alpha}+(1+y)^{\alpha} d y d x .
$$

In this case $\alpha=(\sqrt{\mathbf{1 7}}-3) / 2$.

EXAMPLE 4.3. Another method of constructing a Cantor subset of $[0,1]$ may be described as follows.

Choose a number $u$ from $[0,1]$ with respect to the uniform distribution, then choose $x$ from $[0, u]$ with respect to the uniform distribution on $[0, u]$ and independently choose a number $y$ from $[u, 1]$ with respect to the uniform distribution on $[u, 1]$. Set $J_{0}=[0, x]$ and $J_{1}=[y, 1]$. Continue this process by rescaling to each interval already determined. Again, according to Theorem 1.1, with probability one, we obtain a Cantor set with Hausdorff dimension $\alpha=\sqrt{2}-1$, where

$$
1=\Phi(\alpha)=\int_{0}^{1}\left[\frac{1}{u} \int_{0}^{u} x^{\alpha} d x+\frac{1}{1-u} \int_{u}^{1}(1-y)^{\alpha} d y\right] d u .
$$

This example arose naturally in the course of our study of random homeomorphisms of $[0,1][6]$. In that paper, a measure $P$ is constructed on $H$, the space of orientation preserving homeomorphisms of $[0,1]$, which has the property (Theorem 4.16) of [8] that if $B$ is a Borel subset of $[0,1]$, then

$$
\lambda(B)=\int_{H} \lambda(h(B)) d P(h) .
$$

In particular, if $B$ is the Cantor subset of $[0,1]$ constructed by repeatedly removing the middle half interval, then $\lambda(B)$, the Lebesgue measure of $B$, is zero. Therefore, according to $(*)$ for $P$-a.e. $h, \lambda(h(B))=0$. Example 4.3 shows that for $P$-a.e. $h$, the Hausdorff dimension of the Cantor set $h(B)$ is $\sqrt{2}-1$. The measure $P$ was first studied by Dubins and Freedman [5].

EXAMPLE 4.4. Choose $x$ from $[0,1]$ according to the uniform distribution and then choose $y$ from $[x, 1]$ according to the uniform distribution on $[x, 1]$. Set $J_{0}=$ $[0, x]$ and $J_{1}=[y, 1]$. Continue this procedure by rescaling to each of the intervals already obtained. With probability one, we obtain a Cantor set with Hausdorff dimension $\alpha$, where

$$
1=E\left(T_{1}^{\alpha}+T_{2}^{\alpha}\right)=\int_{0}^{1}\left[x^{\alpha}+\frac{1}{1-x} \int_{x}^{1}(1-y)^{\alpha} d y\right] d x .
$$

In this case $\alpha=(\sqrt{5}-1) / 2$. 
EXAMPLE 4.5. We give a construction to show that the final Cantor set may have the same dimension as the dimension of $J_{\varnothing}$ : Choose the positive integer $n$ with probability $2^{-n}$. Then partition the interval $J_{\varnothing}=[0,1]$ into $2^{n^{2}+n}$ intervals of equal length, set $J_{i}=\left[2 i / 2^{n^{2}+n},(2 i+1) / 2^{n^{2}+n}\right], i=0, \ldots, 2^{n^{2}+n-1}$. Continue this construction. We have

$$
\Phi(\beta)=\sum_{n=1}^{\infty} 2^{-n}\left[\frac{1}{2^{n^{2}+n}}\right]^{\beta} 2^{n^{2}+n-1} .
$$

Thus, $\Phi(\beta)=+\infty$ if $\beta<1$ and $\Phi(1)=\frac{1}{2}$.

EXAMPLE 4.6. We construct at random a Cantor subset of $[0,1] \times[0,1]$ with Hausdorff dimension 1. Choose four numbers $\mu_{1}, \mu_{2}, \mu_{3}, \mu_{4}$ independently from $[0,1]$. Let $x_{1}=\min \left(\mu_{1}, \mu_{2}\right), x_{2}=\max \left(\mu_{1}, \mu_{2}\right), x_{3}=\min \left(\mu_{3}, \mu_{4}\right)$ and $x_{4}=$ $\max \left(\mu_{3}, \mu_{4}\right)$. Let $J_{1}=\left[0, s_{1}\right] \times\left[0, s_{1}\right]$, where $s_{1}=\min \left(x_{1}, x_{3}\right)$, let $J_{2}=\left[1-s_{2}, 1\right] \times$ $\left[0, s_{2}\right]$, where $s_{2}=\min \left(x_{3}, 1-x_{2}\right)$. Let $J_{3}$ be the largest square with one vertex at $(1,1)$ which lies in the rectangle $\left[x_{2}, 1\right] \times\left[x_{4}, 1\right]$. Similarly, let $J_{4}$ be the largest square with one vertex at $(0,1)$ which lies in the rectangle $\left[0, x_{1}\right] \times\left[x_{4}, 1\right]$. Finally, consider the rectangle $\left[x_{1}, x_{2}\right] \times\left[x_{3}, x_{4}\right]$ and its center $\left(\left(x_{1}+x_{2}\right) / 2,\left(x_{3}+x_{4}\right) / 2\right)$. Let $J_{5}$ be the largest square lying in this rectangle and having the same center. Iterate this process. According to our results, with probability one the final object is a Cantor subset of $[0,1] \times[0,1]$ with Hausdorff dimension $\alpha$ where

$$
1=\Phi(\alpha)=5 \iiint \int_{[0,1]^{4}}\left(\mu_{1} \wedge \mu_{2} \wedge \mu_{3} \wedge \mu_{4}\right)^{\alpha} d \mu_{1} d \mu_{2} d \mu_{3} d \mu_{4}
$$

One can check

or

$$
1=\Phi(\alpha)=5\left[4 ! \int_{0}^{1} \int_{0}^{\mu_{4}} \int_{0}^{\mu_{3}} \int_{0}^{\mu_{2}} \mu_{1}^{\alpha} d \mu_{1} d \mu_{2} d \mu_{3} d \mu_{4}\right],
$$

$$
1=\Phi(\alpha)=5 ! /(\alpha+1)(\alpha+2)(\alpha+3)(\alpha+4) .
$$

Obviously, $\alpha=1$.

EXAMPle 4.7 (SIERPIŃSKi UNIVERSAL CURVE). Set $J_{\varnothing}=[0,1] \times[0,1]$. Choose $x_{1}, x_{2}, x_{3}$, and $x_{4}$ from $\left(\frac{1}{3}, \frac{1}{2}\right)$ independently and each according to the uniform distribution. Set $J_{1}=\left[0, x_{1}\right] \times\left[0, x_{1}\right], J_{2}=\left[1-x_{2}, 1\right] \times\left[0, x_{2}\right], J_{3}=\left[0, x_{3}\right] \times\left[1-x_{3}, 1\right]$ and $J_{4}=\left[1-x_{4}, 1\right] \times\left[1-x_{4}, 1\right]$. Since $\frac{1}{3}<x_{1}, x_{2}<\frac{1}{2}$, we have $1-\left(x_{1}+x_{2}\right)<$ $\min \left(x_{1}, x_{2}\right)$. Set $J_{5}=\left[x_{1}, 1-x_{2}\right] \times\left[0,1-\left(x_{1}+x_{2}\right)\right]$. Thus, $J_{5}$ is a square having one side a subset of a side of $J_{1}$ and one side a subset of $J_{2}$. Similarly, let $J_{6}$ be the square having one side in common with $J_{1}$, one side in common with $J_{3}$ and one side on the $Y$-axis. $J_{7}$ is a square similarly placed in relation to $J_{2}$ and $J_{4}$, and $J_{8}$ is similarly placed in relation to $J_{3}$ and $J_{4}$. Thus, the first stage in this construction is analogous to the first stage in the construction of Sierpiński's carpet [17]. Of course, in Sierpiński's construction there is no random element, one simply partitions the unit square into nine congruent subsquares and deletes the middle square. Our construction is more like a Swiss flag:

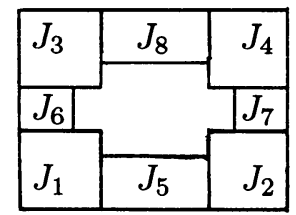


Now, one iterates this process. In order to see that the final set is a topological Sierpiński curve, we use Whyburn's topological characterization [18]. It is easily seen that our final set is locally connected (since for each $\varepsilon>0$, it can be expressed as the union of finitely many subcontinua of diameter $<\varepsilon$ ) and its complement with respect to $S^{2}$, the two-sphere, is the union of countably many open disks $D_{1}, D_{2}, D_{3}, \ldots$ such that for each $i$ and $j$, the boundary of $D_{i}$ is a simple closed curve and if $i \neq j, \bar{D}_{i} \cap \bar{D}_{j}=\varnothing$. These properties characterize Sierpiński's curve.

According to Theorem 1.1, the Hausdorff dimension of the final Sierpiński curve is $\alpha$, where

$$
\begin{aligned}
1 & =\Phi(\alpha)=24 \int_{1 / 3}^{1 / 2} x^{\alpha} d x+144 \int_{1 / 3}^{1 / 2} \int_{1 / 3}^{1 / 2}(1-(x+y))^{\alpha} d y d x \\
& =\frac{24}{\alpha+1}\left[\frac{1}{2^{\alpha+1}}-\frac{1}{3^{\alpha+1}}+\left(\frac{2}{\alpha+2}\right)\left[\frac{1}{3^{\alpha+1}}-\frac{1}{6^{\alpha+1}}\right]\right] .
\end{aligned}
$$

We know that $\Phi(1) \geq 1\left[7\right.$, p. 107] (actually $\Phi(1)=\frac{7}{3}$ and $\Phi(2)<1$ ). Numerical studies show that $\alpha \cong 1.8947$.

EXAMPLE 4.8. Set $J_{\varnothing}=[0,1] \times[0,1] \times[0,1]$. Choose $x_{i}, i=1,2, \ldots, 8$, independently from the open interval $\left(\frac{1}{3}, \frac{1}{2}\right)$ and each according to the uniform distribution. Let $J_{i}$ be a cube lying in $J$ with edge length $x_{i}$, with faces parallel to the coordinate planes and with one vertex at $\left(i_{1}, i_{2}, i_{3}\right)$, where $i=1+i_{1}+i_{2} 2+i_{3} 2^{2}$. Let $J_{9}$ be a cube lying in $J$ with edge length $1-\left(x_{1}+x_{2}\right)$ having one face a subset of a face of $J_{1}$, one face a subset of a face of $J_{2}$, and one edge on the $x$-axis. Continue placing cubes in this manner, until there are a total of twenty cubes. Then iterate this construction inside each of these twenty cubes. The first stage of this construction is somewhat like the first stage in the construction of Menger's universal curve [14, p. 345]. (There is a sketch of this curve in the second edition between pp. 346 and 347.) Of course, in Menger's construction, one partitions the unit cube into 27 congruent subcubes and then one deletes the center cube and the six other cubes having a face in common with it. It is easy to see that the final set $K$ is a locally connected continuum and that every nonempty open subset of $K$ contains $K_{5}$, the complete graph on five vertices and also contains $K_{3,3}$. Thus, our final object is certainly nonplanar. If we knew $K$ were one-dimensional, then we could use R. D. Anderson's result [2, Theorem XII] to conclude that $K$ is a Menger curve, but we have been unable to show this.

According to Theorem 1.1, the Hausdorff dimension of the final universal curve is $\alpha$, where

$$
\begin{aligned}
1 & =\Phi(\alpha)=48 \int_{1 / 3}^{1 / 2} x^{\alpha} d x+432 \int_{1 / 3}^{1 / 2} \int_{1 / 3}^{1 / 2}(1-(x+y))^{\alpha} d y d x \\
& =\frac{48}{\alpha+1}\left[\frac{1}{2^{\alpha+1}}-\frac{1}{3^{\alpha+1}}+\left(\frac{3}{\alpha+2}\right)\left[\frac{1}{3^{\alpha+1}}-\frac{1}{6^{\alpha+1}}\right]\right] .
\end{aligned}
$$

We have $2>\Phi(2)>1$ and $\Phi(3)<1$. Numerical studies show that $\alpha \cong 2.5968$.

REMARK. Our Example 4.7, concerning Sierpiński curves, is in accordance with the category version. Mazurkiewicz showed that for almost all (in the sense of category) continuous maps $f$ of $[0,1]$ into $[0,1] \times[0,1]$, the image $f([0,1])$ is a Sierpiński curve $[\mathbf{1 3}]$. On the other hand, our Example 4.9, concerning Menger 
curves, stands in contrast with the category version for almost all continuous maps $f$ of $[0,1]$ into $[0,1]^{3}$, the image set is an arc $[\mathbf{1 0}]$.

EXAmple 4.9 (Menger Universal CURVE). Let $J_{\varnothing}=[0,1] \times[0,1] \times[0,1]$. Choose $x_{i}, i=1,2, \ldots, 8$, independently from the open inteval $\left(0, \frac{1}{2}\right)$ with common uniform distribution. Construct subcubes $J_{1}, \ldots, J_{8}$ as in Example 4.8. Let $k\left(x_{1}\right.$. $\left.x_{2}\right)$ be the least positive integer greater than $3\left(1-x_{1}-x_{2}\right) /\left(x_{1} \wedge x_{2}\right)$. Now, place $k\left(x_{1}, x_{2}\right)$ nonoverlapping subcubes of $J$ with equal edge length, each with an edge on the $x$-axis and forming a chain from $J_{1}$ to $J_{2}$. Similarly, join each pair of the original eight cubes which are adjacent. This completes the first stage in the construction. Iterate this process in each of the subcubes obtained so far. As in Example 4.8, it is easy to see that $K$ is a locally connected continuum and every nonempty open subset of $K$ is nonplanar. To conclude that $K$ is a Menger curve, we first estimate the Hausdorff dimension of $K$. We have

$$
\Phi(\beta)=16 \int_{0}^{1 / 2} x^{\beta} d x+48 \int_{0}^{1 / 2} \int_{0}^{1 / 2} k\left(x_{1}, x_{2}\right)\left(\frac{1-x_{1}-x_{2}}{k\left(x_{1}, x_{2}\right)}\right)^{\beta} d x_{1} d x_{2}
$$

In particular,

$$
\begin{aligned}
\Phi(2) & \leq \frac{2}{3}+48 \int_{0}^{1 / 2} \int_{0}^{1 / 2} \frac{\left(1-x_{1}-x_{2}\right)^{2}}{k\left(x_{1}, x_{2}\right)} d x_{1} d x_{2} \\
& \leq \frac{2}{3}+48 \int_{0}^{1 / 2} \int_{0}^{1 / 2} \frac{\left(x_{1} \wedge x_{2}\right)}{3}\left(1-x_{1}-x_{2}\right) d x_{1} d x_{2} \\
& \leq \frac{2}{3}+16\left[\int_{0}^{1 / 2} \int_{0}^{x_{2}} x_{1}\left(1-x_{1}-x_{2}\right) d x_{1} d x_{2}\right. \\
& \left.+\int_{0}^{1 / 2} \int_{x_{2}}^{1 / 2} x_{2}\left(1-x_{1}-x_{2}\right) d x_{1} d x_{2}\right] \\
& \leq \frac{2}{3}+32 \int_{0}^{1 / 2} \int_{0}^{x_{2}} x_{1}-x_{1}^{2}-x_{2} x_{1} d x_{1} d x_{2} .
\end{aligned}
$$

So, $\Phi(2) \leq \frac{11}{12}$.

Since $\Phi$ is finite valued, we have that $\operatorname{dim}_{H}(K)=\alpha<2$. This means that the topological dimension of $K$ is $\leq 1$ [9, p. 8]. Thus, with probability one, $K$ has dimension one and according to Anderson's theorem, $K$ is a Menger curve.

\section{REFERENCES}

1. K. B. Athreya and P. E. Ney, Branching processes, Springer-Verlag, Berlin, 1972.

2. R. D. Anderson, A characterization of the universal curve and a proof of its homogeneity, Ann. of Math. (2) 67 (1958), 313-324.

3. 68 (1958), 1-16.

4. J. W. Cannon, Book review, Amer. Math. Monthly 91 (1984), 594-598.

5. L. E. Dubins and D. A. Freedman, Random distribution functions, Proc. Fifth Berkeley Sympos. on Math. Statistics and Probability (L. M. LeCam and J. Neyman, eds.), Univ. of California Press, Berkeley, Calif., 1967, pp. 183-214.

6. P. Doubilet, On the foundations of combinatorial theory. VIII: Symmetric functions through the theory of distribution and occupancy, Stud. Appl. Math. 51 (1972), 377-396.

7. K. J. Falconer, The geometry of fractal sets, Cambridge Tracts in Math., vol. 85, Cambridge Univ. Press, Cambridge, 1985. 
8. S. Graf, R. D. Mauldin and S. C. Williams, Random homeomorphisms, Adv. in Math. (to appear).

9. W. Hurewicz and H. Wallman, Dimension theory, Princeton Math. Series, vol. 4, Princeton Univ. Press, Princeton, N.J., 1941.

10. V. Jarńik, Über die stetigen Abbildungen der Strecke, Monatsh. Math. Phys. 41 (1934), $408-428$.

11. B. B. Mandelbrot, The fractal geometry of nature, Freeman, San Francisco, Calif., 1982.

12. __ Fractals: form, chance and dimension, Freeman, San Francisco, Calif., 1977.

13. S. Mazurkiewicz, Über die stetigen Abbildungen der Strecke, Fund. Math. 25 (1935), 253260.

14. K. Menger, Kurventheorie, Chelsea, New York, 1967.

15. P. A. P. Moran, Additive functions of intervals and Hausdorff measure, Proc. Cambridge Philos. Soc. 42 (1946), 15-23.

16. C. A. Rogers and S. J. Taylor, Functions continuous and singular with respect to a Hausdorff measure, Mathematika 8 (1961), 1-31.

17. W. Sierpiński, Sur une courbe cantorienne qui contient une image biunivoque et continue de toute courbe donée, C. R. Acad. Sci. Paris 162 (1916), 626-631.

18. G. T. Whyburn, Topological characterization of the Sierpiński curve, Fund. Math. 45 (1958), 320-324.

19. U. Zähle, Random fractals generated by random cutouts, Math. Nachr. 116 (1984), 27-52.

Department of Mathematics, North Texas State University, Denton, TeXas 76203-5116

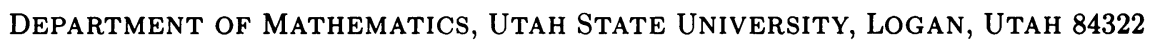

